\title{
Update on Medication-Overuse Headache and Its Treatment
}

\author{
Maria Adele Giamberardino, $M D^{1}$ \\ Dimos-Dimitrios Mitsikostas, $M D^{2}$ \\ Paolo Martelletti, $M D^{3, *}$
}

\author{
Address \\ ${ }^{1}$ Headache Center and Geriatrics Clinic, Department of Medicine and Science of \\ Aging, "G. D’Annunzio" University, via Carlo de Tocco n. 3, 66100, Chieti, Italy \\ ${ }^{2}$ Neurology Department, Athens Naval Hospital, 77A Vas. Sofias Avenue, 11521, \\ Athens, Greece \\ ${ }^{*}, 3$ Department of Clinical and Molecular Medicine, Regional Referral Headache \\ Centre, "Sant'Andrea" Hospital, "Sapienza" University, Via di Grottarossa 1035, \\ 00189, Rome, Italy \\ Email: mag@unich.it
}

Published online: 3 July 2015

(C) Springer Science+Business Media New York 2015

This article is part of the Topical Collection on Headache

Keywords Medication-overuse headache - Chronic migraine - Relapse - Drug withdrawal - Detoxification . Re-prophylaxis · OnabotulinumtoxinA · Topiramate

\section{Opinion statement}

Medication-overuse headache-i.e., a too-frequent consumption of acute headache medications leading to increased headache frequency and reduced effectiveness of acute and preventive treatments-is a serious medical condition whose pathophysiology still remains incompletely known, which is reflected into a lack of mechanism-based treatments. The first mandatory step in the therapeutic strategy remains withdrawal of the abused drug, preferably abrupt, in concomitance with a detoxification pharmacological regimen to lessen withdrawal symptoms. Intravenous hydration, antiemetics, corticosteroids (prednisone), tranquilizers (benzodiazepine), neuroleptics, and rescue medication (another analgesic than the overused) should be delivered in various combinations, on an inpatient (hospitalization and day hospital) basis or outpatient basis, depending on the characteristics of the specific patient and type of overuse. Inpatient withdrawal should be preferred in barbiturate and opioid overuse, in concomitant depression, or, in general, in patients who have difficulty in stopping the overused medication as outpatients. In contrast, in overuse limited to simple analgesics in highly motivated patients, without high levels of depression and/or anxiety, home detoxification should be chosen. Reprophylaxis should immediately follow detoxification, ideally with local injections of onabotulinumtoxinA every 3 months or topiramate orally for at least 3 months. Adequate information to patients about the risks of a too-frequent consumption of symptomatic headache medications is essential and should constantly parallel treatment to help preventing relapse after detoxification and re-prophylaxis. 


\section{Introduction}

Headache is a very frequent medical condition and among the top three causes of disability worldwide [1-4]. Though most headaches are episodic, 2-5\% of the general population experience chronic forms, i.e., headache for $\geq 15$ days per month. Medicationoveruse headache $(\mathrm{MOH})$, i.e., a too-frequent consumption of acute headache medications, develops in approximately $50 \%$ of the patients with chronic headache, although virtually any patient with a primary episodic headache may be at risk of the condition $[3-5,6 \bullet \bullet, 7]$. The estimated world prevalence of $\mathrm{MOH}$ is $0.5-7.2 \%$; women are mainly affected $(\mathrm{M} / \mathrm{F}=1: 4)$ with peak prevalence around 40 years and a decline with age, prevalence reaching 1$1.5 \%$ among people over 65 years. $\mathrm{MOH}$ prevalence is also inversely related to socioeconomic position $[6 \bullet \bullet, 8,9]$.

Migraine is the most common diagnosis leading to $\mathrm{MOH}$ due to overuse of specific antimigraine drugs (triptans and ergot alkaloids] or non-specific drugs [non-steroidal anti-inflammatory drugs (NSAIDs), aspirin, simple analgesics, and combination analgesics], while $\mathrm{MOH}$ developing in tension-type headache is most often caused by NSAID overuse. The mean critical duration of overuse would be shortest for triptans (1.7 years), longer for ergotamine (2.7 years), and longest for simple analgesics (4.8 years) [10-12]. In Europe, $\mathrm{MOH}$ is rarely caused by overuse of opioids, employment of these compounds for symptomatic headache being largely discouraged in the medical environment [13]. Although not recommended as first-line therapy by consensus guidelines, in the USA, opioid analgesics are instead commonly used to treat headaches, and the related medication-overuse represents a significant clinical problem, whose management is particularly complex and usually produces poorer outcomes (see section on "Treatment") [14].

Whatever the medication-overuse headache agent (MOHA), a history of gradual increase in the usage of the specific MOHA is often present, which is then accompanied by increasing frequency of headache with decreasing effectiveness of the individual doses of MOHA in relieving the individual headaches. Reduced effectiveness of preventative treatments is also a typical consequence of $\mathrm{MOH}$ [12]. Most often comorbid with emotional and psychiatric disturbance, such as depression or anxiety, and numerous additional pain and non-pain medical conditions, $\mathrm{MOH}$ produces decreased quality of life and increased disability [15-23].

"Headache induced by chronic substance use or exposure" was already described in the 1988 International Headache Society (IHS) Headache Classification; the term $\mathrm{MOH}$, however, only appeared in the 2004 edition, where MOH was defined as probable, until its improvement after detoxification/ withdrawal of medication allowed a definite diagnosis $[6 \bullet \bullet, 24,25]$. The last HIS criteria published in 2013 define $\mathrm{MOH}$ as headache manifesting on $\geq 15$ days/month in a patient with a preexisting headache disorder, developing as a consequence of regular overuse of acute or symptomatic headache medication (on $\geq 10$ or $\geq 15$ days/month, depending on the medication) for more than 3 months, which usually, but not constantly, resolves after interruption of the overuse (Table 1) [26, 27]. With these criteria, $\mathrm{MOH}$ can be diagnosed immediately and independently of withdrawal.

The pathophysiology of the condition is still partially unknown. After medication overuse, an increased excitability has been demonstrated of neurons in the cerebral cortex, which may facilitate the occurrence of cortical spreading depression, and trigeminal system, which may promote phenomena of peripheral and central sensitization. These changes are probably the result of alterations in several modulating systems (e.g., serotonin, endocannabinoid dependent) at the central level, involved in pain perception in genetically predisposed individuals $[11,28 \bullet]$.

There are currently no internationally accepted guidelines for the treatment of $\mathrm{MOH}$ and protocol strategies vary greatly among centers $[5,6 \bullet \bullet]$. The most frequent therapeutic steps are abrupt or gradual withdrawal of the abused drug, an inpatient or outpatient detoxification protocol regimen, and re-prophylaxis, generally with onabotulinumtoxinA or topiramate, to prevent relapse, whose risk is highest in the first 12 months [12]. 


\section{Table 1. Medication-overuse headache}

Diagnostic criteria:

A. Headache occurring on $\geq 15$ days per month in a patient with a preexisting headache disorder

B. Regular overuse for $>3$ months of one or more drugs that can be taken for acute and/or symptomatic treatment of headache for $\geq 10$ days/month, ergotamine, triptans, opioids, combination analgesics, multiple drug classes not individually overused, unverified overuse of multiple drug classes, and one or more medications other than those described above and below

for $\geq 15$ days/month, simple analgesics: paracetamol or acetylsalicylic acid or other non-steroidal anti-inflammatory drug (NSAID) (one or more NSAIDs other than acetylsalicylic acid)

C. Not better accounted for by another ICHD-3 diagnosis

ICHD-3 beta 2013

\section{Treatment}

\section{General considerations for treatment protocols}

Due to pathophysiological uncertainty, there is still a lack of mechanism-based therapies and, as already stated in the "Introduction", specific guidelines for the management of $\mathrm{MOH}$ are currently not available $[5,6 \bullet \bullet]$. Regardless of the strategy, however, the scientific community agrees that the main aims of the treatment are as follows: (i) Withdrawal of the overused drug leading to detoxification(s), (ii) pharmacological and non-pharmacological support provided to patients, and (iii) prevention of relapse.

In all patients, independently of the type of abuse, particular attention should be paid to the presence of comorbidities, especially psychiatric, e.g., depression, anxiety, and bipolar disorder, in regard to the modalities of withdrawal, and type of support during detoxification and in the follow-up period $[8,21]$.

Withdrawal of the overused drug/s is the first mandatory approach since this in most cases leads to an improvement of the headache. However, there are still many debated areas with this respect, for instance, if withdrawal should be abrupt or gradual, which protocol of support should be given to patients during withdrawal, which is the preferred setting during detoxification, i.e., inpatient versus outpatient care, or whether or not prophylactic medication should be initiated immediately at withdrawal or after withdrawal therapy has been completed.

Some studies seem to indicate that simple advice to withdrawal is effective. For example, in patients affected with $\mathrm{MOH}$ for 8-18 years, a population-based study carried out in Norway showed a notable improvement subsequent to simple information about the use of medication: $42 \%$ of the patients reverted to episodic headache and $76 \%$ were free of medication overuse after 1.5 years [29]. This study, however, was observational and lacked a control group. 
Further studies by Italian neurologists also showed the efficacy of simple advice in reverting $\mathrm{MOH}$ at 2 months in $78-92 \%$ of patients with simple $\mathrm{MOH}$ and $60 \%$ of patients with complicated $\mathrm{MOH}[30,31]$.

A recent paper by Munksgaard and Jensen [32 $\bullet$ reviewing the literature on $\mathrm{MOH}$ treatment also suggests that simple detoxification and information can reduce headache frequency to episodic headache in over $50 \%$ of the patients, with approximately half of the patients not needing prophylactic treatment after withdrawal. Also, pain hypersensitivity, typically present in $\mathrm{MOH}$ and indicating central sensitization, reverts in patients successfully detoxified, indicating a reversible mechanism at the basis of the condition. However, other authors report much less favorable outcomes with simple withdrawal and advice and are thus in favor of a more robust support to patients during detoxification. In this phase, in fact, the majority of the patients present withdrawal symptoms for 2-10 days, i.e., rebound headache (initial worsening of the headache), nausea, vomiting, hypotension, tachycardia, restlessness, anxiety, nervousness, and sleep disturbances. Withdrawal headaches have different duration, depending on the drugs causing the overuse, i.e., it is shorter in patients overusing triptans (4.1 days) than in those overusing ergotamine (6.7 days) or NSAIDs/ analgesics ( 9.5 days) $[5,6 \bullet \bullet$. Withdrawal from drugs containing barbiturates and antimigraine compounds may also produce seizures or hallucinations. Various drug classes can be used to lessen/eliminate these symptoms, although detoxification drug protocols differ greatly among different centers (see specific sections below). Different settings have been suggested for successful withdrawal/detoxification: home treatment, hospitalization, or day hospital. Inpatient withdrawal (2 days-2 weeks) seems the most effective (reported success around $70 \%$ ) and it should be the preferred approach in patients with barbiturate and opioid overuse, depressed patients, or, in general, patients who have difficulty in stopping the overused medication as outpatients. In contrast, highly motivated patients, without high levels of depression and/or anxiety, in whom the overuse is limited to simple analgesics or analgesics not containing barbiturates or opioids better benefit from an outpatient detoxification approach [13]. A day-hospital regimen is also useful, with patients remaining in the hospital for no longer than $6 \mathrm{~h}$ during pharmacological infusion treatment [12]. Abrupt versus gradual suspension of the abused drug is another point of debate. There are currently no studies directly comparing the results of abrupt versus gradual suspension; however, the clinical experience suggests that in most cases, abrupt interruption achieves better results as it probably promotes a fast resolution of the drug-induced pain-coping behavior [33]. Abrupt discontinuation can be performed with overuse of triptans, ergots, paracetamol, aspirin, and NSAIDs, while barbiturates, opioids, and benzodiazepines, unless replaced with long-acting derivatives, must be tapered to avoid a serious withdrawal syndrome. A gradual reduction is also preferred with compounds containing caffeine [13].

The role of re-prophylaxis is still controversial. Prophylactic treatment with onabotulinumtoxinA and topiramate in chronic migraine with $\mathrm{MOH}$ (see below) has proven effective in placebo-controlled studies, with a significant reduction of headache days per month [34-37]. However, these results have proven not superior to detoxification without prophylactic treatment in some 
studies $[29,38]$. On this basis, a number of authors believe that while initial withdrawal is the treatment of choice in $\mathrm{MOH}$, prophylaxis should be restricted to patients who do not obtain sufficient results from withdrawal [30]. Since it is still not possible to predict in advance the response/lack of response to withdrawal in the single patient, however, other authors are in favor of prophylaxis to be applied routinely in every case, to optimize the outcome. Whether prophylaxis should be started immediately at withdrawal or after completion of the withdrawal process remains debated [39•].

The follow-up of patients subjected to detoxification has shown controversial results. The short-term response (2-month follow-up) to drug withdrawal in $\mathrm{MOH}$ has been suggested to be genetically determined [40]. Regarding relapse, recent studies indicate that the highest risk is in the first 12 months, although different percentages are reported by various authors $(22-44 \%$ in Trucco et al. [41]; 60-75 \% in Zidverc et al. [42] and Baumgartner et al. [43]; or 40-60 \% in Andrasik [15], Katsarava [44], Hagen et al. [45], and Fritsche et al. [46]). The risk decreases, however, if medication overuse is avoided for 12 months after withdrawal, and in general, $\mathrm{MOH}$ treatment is considered successful if the improvement obtained after withdrawal/detoxification with or without prophylaxis is maintained at 1-year follow-up [42, 47-51]. Several factors are considered a risk for relapse: male sex, a diagnosis of tension-type headache or mixed headache, frequency of the primary headache, higher severity of the migraine condition, long duration of drug overuse, greater number of previous prophylactic treatments, intake of combination analgesic products (e.g., one or more NSAIDs with caffeine or codeine) or of codeine-containing drugs, ergotamine or triptan withdrawal more than analgesic withdrawal, use of the same drug that has caused overuse, lower improvement after drug withdrawal, reduced sleep quality, and high levels of body pain and of disability score for chronic headache [12]. Psychiatric comorbidity has also been suggested to be a predictor of reduced response to treatment $[13,52]$. Pharmacogenomics studies will hopefully allow the medical community to be involved in headache treatment to better predict the outcome of drug treatment and thus help in managing the condition. The long-term prognosis in $\mathrm{MOH}$ has been investigated in lesser detail with respect to the short-term outcome. Prospective studies that included patients with triptan-induced $\mathrm{MOH}$ have reported relapse rates after successful drug detoxification therapy of $38 \%$ in the first year and $42 \%$ after 4 years [44, 47]. Patients with tension-type headache had higher relapse rates than migraine patients, and analgesic overusers had higher relapse rates than ergot and triptan overusers [51]. Results on long-term relapse in the literature should, however, be compared with some caution, since the studies varied in the use of different headache classification systems, different withdrawal and prophylaxis, and follow-up and criteria for improvement. Furthermore, the percentages of relapse with the same MOHA versus relapse with a different MOHA in the same patients still remain to be precisely determined with appropriately designed investigation [5].

Prevention represents the most crucial aspect for $\mathrm{MOH}$, and numerous studies underline the key role played by information to the headache 
patients about the risk of the overuse of medications [53]. The major steps in the treatment approach to $\mathrm{MOH}$ are summarized in Table 2.

\section{Pharmacological treatment}

Drug therapy is aimed at reducing withdrawal symptoms during the detoxification phase, at preventing subsequent relapse (re-prophylaxis), and then at stabilizing the residual headache (standard long-term treatment).

\section{Detoxification/Rehabilitation}

Pharmacological protocols for detoxification/rehabilitation are highly variable in the various centers. The following are most frequently used in the majority of the patients: intravenous hydration, corticosteroids, antiemetics, tranquilizers, neuroleptics, and rescue medication (another analgesic than the overused). Intravenous dihydroergotamine (DHE) is also used specifically in inpatient protocols in the very first phases ( 4 days, every $8 \mathrm{~h}$ ) of the detoxification process, in combination with an antiemetic to control nausea, which is the most frequent side effect. It has been shown to be efficacious, shortening hospital stay and reducing analgesic withdrawal symptoms. Due to the temporarily effect of blood vessel vasoconstrictions, DHE is, however, contraindicated in patients with a past history of cardiovascular disease or stroke [13].

Regarding corticosteroids, oral prednisone represents the most common treatment during detoxification [12]; when compared with placebo, it reduces the duration of withdrawal headache [33, 54-56]. The use of prednisolone has instead produced mixed results; in particular, it has been found to be not superior to placebo in two placebo-controlled studies [57, 58].

As a general rule, steroids should be employed with particular caution in patients with significant comorbidities, considering the long list of their contraindications. The risk of steroid psychosis furthermore needs to be taken into account during acute treatment in the detoxification phase, with immediate suspension of the drug, should any symptoms of the condition appear, e.g., hallucinations, combined to eventual therapy with phenothiazines [13].

Recently, a double-blind, parallel-group, clinical trial by Taghdiri et al. [59] compared the efficacy of celecoxib ( $400 \mathrm{mg} /$ day for the first 5 days and then decreased at a rate of $100 \mathrm{mg}$ every 5 days) versus prednisone, orally $(75 \mathrm{mg} /$ day

\section{Table 2. Therapeutic steps in $\mathrm{MOH}$}

- Educate the patient on $\mathrm{MOH}$ and obtain assent for treatment

- Withdrawal or detoxification

- Treat the headaches associated with detoxification

- Use appropriate prophylactic antimigraine agents either early or later after detoxification

- Treat residual headache

- Provide appropriate psychiatric support when necessary

- Be prepared for longer term follow-up to help the patient from relapsing into $\mathrm{MOH}$ again

Source: Couch and Lenaerts, 2007 
for the first 5 days and then tapered off every 5 days) in $\mathrm{MOH}$. In the celecoxib group, headache intensity was lower than in the prednisone group during the first 3 weeks after withdrawal, although headache frequency and the need for rescue medication intake were not different between the two groups. The authors conclude that the use of celecoxib and prednisone is equivalent toward the reduction of headache days or rescue medication intake during withdrawal in $\mathrm{MOH}$.

Whatever the main drug used in the detoxification protocol, antiemetics, such as promethazine, hydroxyzine, or metoclopramide, should systematically be delivered $[5,13]$.

A typical inpatient protocol adopted at eight Italian hospitals provided satisfactory results at 6-month follow-up in patients with probable chronic migraine and chronic $\mathrm{MOH}$. It involved abrupt discontinuation of the overused drug and therapy with i.v. hydration, steroids (dexamethasone, $4 \mathrm{mg}$ i.v./day for 1 week), and benzodiazepines (diazepam $6 \mathrm{mg} /$ day for 10 days) along with metoclopramide and early prophylaxis (initiated soon after hospital admission) [60].

Re-prophylaxis

Topiramate and local injection of onabotulinumtoxinA are the compounds with the best evidence of efficacy as therapeutic agents for re-prophylaxis after detoxification in patients with chronic migraine with and without overuse. Topiramate has been proven effective in decreasing the number of migraine days in multicenter, randomized, double-blind, placebo controlled trials [6163], and onabotulinumtoxinA has also significantly reduced headache pain in $\mathrm{MOH}$ associated with chronic migraine [64].

The comparative efficacy and safety of these two approaches for chronic migraine were evaluated in two studies $[65,66]$. Both drugs proved equally effective in improving several pain/disability parameters, but the safety profile was higher for onabotulinumtoxinA and the discontinuation rate significantly higher for topiramate [adverse events (AEs) being the major reason for withdrawal] (discontinuation rates 25-44.2\% for topiramate; $10-25 \%$ for onabotulinumtoxinA) [12, 65-69].

These results indicate that onabotulinumtoxinA is an important option for re-prophylaxis in chronic migraine associated with $\mathrm{MOH}$.

Sodium valproate and amitriptyline have also been recently tested in reprophylaxis of $\mathrm{MOH}$, although more studies are needed with these compounds to confirm the results $[70 \bullet, 71]$.

The phase of re-prophylaxis is particularly critical, as it regards the immediate post-detoxification period, where the risk of relapse is highest. This phase should preferentially be handled by the same medical staff that has taken care of detoxification, with the physician being ready to help in the case of relapse [72, 73].

\section{Topiramate}

- Topiramate acts through different mechanisms: inhibitory effects on voltage-gated sodium and calcium channels, modulation of glutamatemediated neurotransmission, enhancement of some gammaaminobutyric acid (GABA) A receptors, and inhibition of carbonic 
anhydrase. It reduces excitatory neurotransmission and enhances inhibitory neurotransmission.

Dose $25 \mathrm{mg} /$ day orally for a week and then increase by $25 \mathrm{mg} /$ day every week till $100 \mathrm{mg} /$ day (50 mg×2/day) to be maintained for 3 months $100 \mathrm{mg} /$ day orally for 3 months

Contraindications Depression, glaucoma, liver and kidney disease, hypokalemia, acidosis, and pregnancy

Main interactions Alcohol, CNS depressant drugs, and contraceptives

Side effects/AE Paresthesias (8.0\%), cognitive symptoms (difficulty with concentration/ attention and memory) $(7.3 \%)$, fatigue ( $4.7 \%)$, insomnia (3.4\%), nausea $(2.3 \%)$, loss of appetite, anxiety, permanent reduction of visual acuity, and dizziness $(2.1 \%)$

Paresthesia, due to the inhibition of carbonic anhydrase, is the most common side effect (which can be lessened by potassium supplementation, e.g., $48 \mathrm{mEq} /$ day) although it does not constitute the main reason for therapy discontinuation. The majority of AEs appear during the titration period, generally within 6 weeks, suggesting that in the case that patients do not present AEs by the end of such period, they will by safe from such events $[56,60]$.

\section{OnabotulinumtoxinA}

Contraindications

Main drug interactions

Side effects/adverse events

- This neurotoxin blocks neuromuscular transmission by binding to acceptor sites on motor or sympathetic nerve terminals, entering the nerve terminals, and inhibiting the release of acetylcholine, a neurotransmitter at somatic neuromuscular junctions, at the parasympathetic nervous system, at sympathetic preganglionic fibers (cholinergic fibers), and at some synapses in the central nervous system. In addition to the well-documented myorelaxant effects, onabotulinumtoxinA can have a direct analgesic effect that likely involves inhibition of primary and secondary nociceptive neurons [74].

Dose 155 units $(1 \mathrm{ml} / 100$ Units), subcutaneously or intramuscularly, divided across 31 points, seven specific head and neck muscles (frontalis, corrugator, procerus, occipitalis, temporalis, trapezius, and cervical paraspinal muscle group) fixed sites fixed doses (FSFD) protocol, [all muscles injected bilaterally with the exception of the procerus muscle which should be injected at one site (midline)] plus, eventually, "Follow The Pain (FTP) protocol" (40 units/8 points), administered at intervals of at least 12 weeks [75].

Infection at the proposed injection site(s) and hypersensitivity to any botulinum toxin preparation or ingredient in the formulations

Aminoglycosides (e.g., gentamicin), drugs interfering with neuromuscular transmission (e.g., quinidine) (the effects of onabotulinumtoxinA may be potentiated), anticholinergic agents (e.g., atropine) (systemic anticholinergic effects, .e.g., blurred vision, may be potentiated), and muscle relaxants (excessive weakness)

Neck pain (9\%), muscle weakness (5.5\%), and headache (5\%). Severe worsening of migraine requiring hospitalization has been reported in approximately $1 \%$ of patients, usually occurring within the first week of treatment (vs placebo, $0.3 \%$ ). Other effects include eyelid ptosis, 
musculoskeletal stiffness, myalgia, musculoskeletal pain and muscle spasms, and injection site pain. No distant spread of toxin effect has been reported at doses recommended for migraine prophylaxis $[35,36,76]$.

\section{Sodium valproate}

Sodium valproate, the acid salt of valproic acid, has anticonvulsant effects mainly due to the blockade of voltage-dependent sodium channels and increased brain levels of GABA. A recent multicenter, randomized, double-blind, placebocontrolled trial (SAMOHA) has investigated the effects of sodium valproate versus placebo [the study randomized $88 \mathrm{MOH}$ patients for a 3-month treatment period with sodium valproate (VPA) $(800 \mathrm{mg} /$ day) or placebo after a 6 -day outpatient detoxification regimen] in the short-term treatment of $\mathrm{MOH}$ after detoxification in patients with a history of migraine without aura, showing the efficacy and safety of this therapeutic approach [70 $]$. The 3-month responder rate (the proportion of patients achieving $\geq 50 \%$ reduction in the number of days with headache per month) was, in fact, $23.8 \%$ for the placebo arm and $45 \%$ for the VPA arm; however, after a 3-month follow-up period, this rate did not differ between the two groups. In a subsequent ancillary study, a further analysis of the data from this trial, on both the placebo and active groups, furthermore showed that in $\mathrm{MOH}$ patients with a history of migraine longer than 30 years, there was no benefit from detoxification and advice to withdraw from drug abuse without preventive therapy. This lack of benefit could be due to the lingering central sensitization underlying the pathophysiology of $\mathrm{MOH}$ [39•].

Dose $800 \mathrm{mg} /$ day orally for 3 months

Contraindications Preexisting liver and pancreatic disease/dysfunction and pregnancy

Main drug interactions Alcohol and benzodiazepines (CNS depressant effects may be potentiated), other antiepileptics, anticoagulants, aspirin, erythromycin, carbapenem, and cimetidine

Side effects/adverse events Digestive symptoms (diarrhea, nausea, and vomiting), vision problems, hormonal disturbances (e.g., menstrual irregularities in women), weight gain, memory problems, hair loss, dizziness, tremor, drowsiness, infections, and low platelet count. Less common but more serious: liver and pancreatic damage, movement disorders, and psychiatric/neurological disturbances (hallucinations, confusion, and anxiety)

\section{Amitriptyline}

Amitriptyline, a tricyclic antidepressant, acts primarily as a serotoninnorepinephrine re-uptake inhibitor, with strong actions on the serotonin transporter and moderate effects on the norepinephrine transporter.

An open-label design study by Fan et al. [71] evaluated the long-term efficacy of low-dose amitriptyline in combination with abrupt withdrawal for $\mathrm{MOH}$ in an outpatient setting for a 1-year observational period. Headache frequency and drug consumption at 3 and 12 months were significantly reduced with respect to baseline, with $58 \%$ of the patients considered as responders at 12 months, i.e., with $\geq 50 \%$ reduction in headache frequency from baseline and being without medication overuse. The authors conclude that early introduction of low-dose amitriptyline combined with abrupt withdrawal could be considered as a choice for patients with $\mathrm{MOH}$, particularly indicated in the case of comorbidity with fibromyalgia. 
Dose $10 \mathrm{mg} /$ day orally for 3 months

Contraindications Pregnancy, history of myocardial infarction and arrhythmias, ischemic heart disease and congestive heart failure, severe liver disease, and concomitant use of monoamine oxidase inhibitors (MAO)

Main drug interactions $\mathrm{MAO}$ (potential risk of serotonin syndrome), fluoxetine, anticholinergic agents (atropine), antipsychotics, analgesics, antithyroid medication and thyroid hormones, cimetidine, and serotoninergic agents (SSRIs and triptans due to the potential for serotonin syndrome)

Side effects Dizziness, weight gain, delirium and confusion, anxiety and agitation, orthostatic hypotension, loss of libido, impotence, sleep disturbances, and drowsiness

\section{Standard long-term treatment}

There is normally an underlying headache problem which will have led to the increasing use of symptomatic headache therapy that resulted in $\mathrm{MOH}[72,73]$. After successful detoxification, this underlying headache is usually still present and must be appropriately managed. Thus, once the immediate post-detoxification phase is over and re-prophylaxis has successfully avoided relapse, a standard re-treatment of the residual headache should be initiated. For treatment of the acute attack, a drug different from the one previously causing the abuse should be preferred, eventually associated with an antiemetic in the case of significant nausea/vomiting in migraine patients. If criteria for prophylaxis are still present, a standard preventative treatment should also be carried out-in cycles of 3 months-according to international guidelines, i.e., for migraine with beta-blockers or calcium channel blockers or antiepileptics/ antidepressants, depending on the profile of the patient and the specific comorbidities and for tension-type headache with antidepressants or benzodiazepines [77-79]. Although in the long run, patients are not necessarily followed by the same medical staff that has dealt with the detoxification process, a stable and longer term relationship with the physician who handled the $\mathrm{MOH}$ therapy usually proves to be very helpful for stabilizing the results $[72,73]$.

\section{Interventional procedures}

Neurostimulation is becoming increasingly employed in the treatment of chronic pain [80]; occipital nerve stimulation (ONS), in particular, is providing encouraging results in refractory chronic migraine [1]. ONS, obtained through an implanted device, is a relatively safe technique compared to other invasive procedures, such as hypothalamic deep brain stimulation. The most frequent adverse events are lead migration, local immediate or delayed infections, and battery depletion due to high stimulation intensities needed to obtain an optimal nerve stimulation in some patients. Patients also complain of unpleasant traction on the connecting cables and sometimes do not tolerate the paresthesias induced by the stimulation of the occipital nerves. ONS probably acts 


\section{Conclusions}

through a non-specific neuromodulatory effect on central pain control systems. Recommendations by the European Headache Federation [81, 82] state that from a medical standpoint, the application of a neurostimulator, either in a trial or on the basis of a CE mark treatment, should be considered only once all alternative drug and behavioral therapies as recommended by international guidelines have failed and medication-overuse headache is excluded. However, whether to include or exclude patients suffering from $\mathrm{MOH}$ is currently being debated and it is possible that future guidelines will reconsider this option also for $\mathrm{MOH}$ [4]. In the meanwhile, a recent trial has indeed evaluated the neurostimulation in chronic refractory headache without excluding $\mathrm{MOH}$, finding positive results, thus suggesting that $\mathrm{MOH}$ could benefit from this interventional option in selected cases. Further studies should be conducted in the field to definitively assess this aspect.

A standardized therapeutic approach to medication-overuse headache is lacking at the present time. The results of the current management options, in terms of immediate success of detoxification, as well as of prevention of relapse, are still unsatisfactory, and further studies investigating the pathophysiology of the condition, combined with clinical trials to test specific medications/protocols, are required to hopefully achieve an internationally agreed standard of care for the condition. While waiting for treatment guidelines, the importance of prevention should be stressed in every possible medical context-from general practice to specialistic context/hospital settings-by adequately informing headache patients about the risks related to overuse of symptomatic medications for their attacks [12, 83].

\section{Compliance with Ethics Guidelines}

\section{Conflict of Interest}

Maria Adele Giamberardino declares the receipt of grants from Epitech Group and of personal fees from Helsinn Healthcare, outside the submitted work.

Dimos-Dimitrios Mitsikostas declares the receipt of consulting fees from Genzyme and Genesis, outside the submitted work.

Paolo Martelletti declares the receipt of grants from Allergan, ACRAF, and Pfizer, as well as personal fees from Bayer, outside the submitted work.

\section{Human and Animal Rights and Informed Consent}

This article does not contain any studies with human or animal subjects performed by any of the authors. 
Papers of particular interest, published recently, have been highlighted as:

- Of importance

$\bullet \quad$ Of major importance

1. Martelletti P. The therapeutic armamentarium in migraine is quite elderly. Expert Opin Drug Metab Toxicol. 2015;11:175-7.

2. Giamberardino MA, Martelletti P. Emerging drugs for migraine treatment. Expert Opin Emerg Drugs. 2015;20:137-47.

3. Global Burden of Disease Study 2013 Collaborators. Global, regional, and national incidence, prevalence, and years lived with disability for 301 acute and chronic diseases and injuries in 188 countries, 19902013: a systematic analysis for the Global Burden of Disease Study 2013. Lancet. 2015. doi:10.1016/S01406736(15)60692-4.

4. Steiner TJ, Birbeck GL, Jensen RH, Katsarava Z, Stovner LJ, Martelletti P. Headache disorders are third cause of disability worldwide. J Headache Pain 2015;16:58. doi:10. 1186/s10194-015-0544-2.

5. Lionetto L, Negro A, Palmisani S, Gentile G, Del Fiore MR, Mercieri M, et al. Emerging treatment for chronic migraine and refractory chronic migraine. Expert Opin Emerg Drugs. 2012;17:393-406.

6.• Kristoffersen ES, Lundqvist C. Medication-overuse headache: epidemiology, diagnosis and treatment. Ther Adv Drug Saf. 2014;5:87-99.

Comprehensive overview of the major aspects of $\mathrm{MOH}$ according to recent criteria.

7. Martelletti P, Katsarava Z, Lampl C, Magis D, Bendtsen L, Negro A, et al. Refractory chronic migraine: a consensus statement on clinical definition from the European Headache Federation. J Headache Pain. 2014;15:47. doi:10.1186/1129-2377-15-47.

8. Westergaard ML, Glümer C, Hansen EH, Jensen RH. Prevalence of chronic headache with and without medication overuse: associations with socioeconomic position and physical and mental health status. Pain. 2014;155:2005-13.

9. Westergaard ML, Hansen EH, Glumer C, Olesen J, Jensen RH. Definitions of medication-overuse headache in population-based studies and their implications on prevalence estimates: a systematic review. Cephalalgia. 2014;34:409-25.

10. Gentile G, Missori S, Borro M, Sebastianelli A Simmaco M, Martelletti P. Frequencies of genetic polymorphisms related to triptans metabolism in chronic migraine. J Headache Pain. 2010;11:151-6.

11. Gentile G, Borro M, Lala N, Missori S, Simmaco M, Martelletti P. Genetic polymorphisms related to efficacy and overuse of triptans in chronic migraine. J Headache Pain. 2010;11:431-5.
12. Negro A, Martelletti P. Chronic migraine plus medication overuse headache: two entities or not? J Headache Pain. 2011;12:593-601.

13. Paemeleire K, Crevits L, Goadsby PJ, Kaube H. Practical management of medication-overuse headache. Acta Neurol Belg. 2006;106:43-51.

14. Mazer-Amirshahi M, Dewey K, Mullins PM, van den Anker J, Pines JM, Perrone J, et al. Trends in opioid analgesic use for headaches in US emergency departments. Am J Emerg Med. 2014;32:1068-73.

15. Andrasik F, Grazzi L, Usai S, Kass S, Bussone G. Disability in chronic migraine with medication overuse: treatment effects through 5 years. Cephalalgia. 2010;30:610-4.

16. Innamorati $M$, Pompili $M$, Erbuto D, Ricci F, Migliorati M, Lamis DA, et al. Psychometric properties of the stagnation scale in medication overuse headache patients. J Headache Pain. 2015;16:2. doi:10.1186/11292377-16-2.

17. Raggi A, Schiavolin S, Leonardi M, Giovannetti AM, Bussone $\mathrm{G}$, Curone $\mathrm{M}$, et al. Chronic migraine with medication overuse: association between disability and quality of life measures, and impact of disease on patients' lives. J Neurol Sci. 2015;348:60-6.

18. Giamberardino MA, Tafuri E, Savini A, Fabrizio A, Affaitati G, Lerza R, et al. Contribution of myofascial trigger points to migraine symptoms. J Pain. 2007;8:869-78.

19. Giamberardino MA, Affaitati G, Fabrizio A, Costantini R. Myofascial pain syndromes and their evaluation. Best Pract Res Clin Rheumatol. 2011;25:185-98.

20. Giamberardino MA, Tana C, Costantini R. Pain thresholds in women with chronic pelvic pain. Curr Opin Obstet Gynecol. 2014;26:253-9.

21. Pompili M, Di Cosimo D, Innamorati M, Lester D, Tatarelli R, Martelletti P. Psychiatric comorbidity in patients with chronic daily headache and migraine: a selective overview including personality traits and suicide risk. J Headache Pain. 2009;10:283-90.

22. Tana C, Santilli F, Martelletti P, di Vincenzo A, Cipollone F, Davì G, et al. Correlation between migraine severity and cholesterol levels. Pain Pract. 2014. doi:10.1111/papr.12229.

23. Tana C, Tafuri E, Tana M, Martelletti P, Negro A, Affaitati $G$, et al. New insights into the cardiovascular risk of migraine and the role of white matter hyperintensities: is gold all that glitters? J Headache Pain. 2013;14:9.

24. Headache Classification Committee of the International Headache Society. Classification and diagnostic 
criteria for headache disorders, cranial neuralgias and facial pain. Cephalalgia. 1988;8 Suppl 7:1-96.

25. Headache Classification Subcommittee of the International Headache Society. The international classification of headache disorders, 2 nd edition. Cephalalgia. 2004;24:1-150.

26. Headache Classification Committee of the International Headache Society (IHS). The international classification of headache disorders, 3rd edition (beta version). Cephalalgia. 2013;33:629-808.

27. Treede RD, Rief W, Barke A, Aziz Q, Bennett MI, Benoliel R, et al. A classification of chronic pain for ICD-11. Pain. 2015: 156(6):1003-7

28. Srikiatkhachorn A, le Grand SM, Supornsilpchai W, Storer RJ. Pathophysiology of medication overuse headache-an update. Headache. 2014;54:204-10.

Comprehensive and critical assessment of pathophysiological hypotheses about $\mathrm{MOH}$ generation.

29. Grande R, Aaseth K, Benth J, Lundqvist C, Russell M. Reduction in medication-overuse headache after short information. The Akershus study of chronic headache. Eur J Neurol. 2011;18:129-37.

30. Rossi P, Di L, Faroni J, Cesarino F, Nappi G, Di Lorenzo C. Advice alone vs. structured detoxification programmes for medication overuse headache: a prospective, randomized, open label trial in transformed migraine patients with low medical needs.

Cephalalgia. 2006;26:1097-105.

31. Rossi P, Faroni J, Tassorelli C, Nappi G. Advice alone versus structured detoxification programmes for complicated medication overuse headache $(\mathrm{MOH})$ : a prospective, randomized, open-label trial. J Headache Pain. 2013;14:10.

32. Munksgaard SB, Jensen RH. Medication overuse headache. Headache. 2014;54:1251-7125.

Comprehensive update on current treatment options for $\mathrm{MOH}$.

33. Obermann M, Katsarava Z. Management of medication-overuse headache. Expert Rev Neurother. 2007;7:1145-55.

34. Diener H, Dodick D, Goadsby P, Bigal M, Bussone G, Silberstein $S$, et al. Utility of topiramate for the treatment of patients with chronic migraine in the presence or absence of acute medication overuse. Cephalalgia. 2009;29:1021-7.

35. Dodick D, Turkel C, Degryse R, Aurora S, Silberstein S, Lipton $\mathrm{R}$, et al. Onabotulinumtoxin for treatment of chronic migraine: pooled results from the doubleblind, randomized, placebo-controlled phases of the preempt clinical program. Headache. 2010;50:921-36.

36. Aurora SK, Dodick DW, Turkel CC, DeGryse RE, Silberstein SD, Lipton RB, et al. OnabotulinumtoxinA for treatment of chronic migraine: results from the double-blind, randomized placebo-controlled phase of the PREEMPT 1 trial. Cephalalgia. 2010;30:793-803.

37. Sandrini G, Perrotta A, Tassorelli C, Torelli P, Brighina $F$, Sances $G$, et al. Botulinum toxin type-A in the prophylactic treatment of medication overuse headache: a multicenter, double-blind, randomized, placebo-controlled, parallel group study. J Headache Pain. 2011;12:427-33.

38. Munksgaard S, Bendtsen L, Jensen R. Detoxification of medication-overuse headache by a multidisciplinary treatment programme is highly effective: a comparison of two consecutive treatment methods in an open-label design. Cephalalgia. 2012;32:834-44.

39. Caproni S, Bianchi E, Cupini LM, Corbelli I, Beghi E, Calabresi $\mathrm{P}$, et al. Long term headache duration is a factor predicting nonresponse to detoxification and advice in medication overuse headache. J Headache Pain. 2014;15:88.

Interesting paper addressing the crucial issue of predictive factors of response/nonresponse to detoxification in $\mathrm{MOH}$.

40. Cargnin S, Viana M, Sances G, Bianchi M, Ghiotto N, Tassorelli $\mathrm{C}$, et al. Combined effect of common gene variants on response to drug withdrawal therapy in medication overuse headache. Eur J Clin Pharmacol. 2014;70:1195-202.

41. Trucco M, Meineri P, Ruiz L, Gruppo Neurologico Ospedaliero Interregionale per lo Studio delle Cefalee (Neurological Hospital Interregional Group for the Study of Headaches). Preliminary results of a withdrawal and detoxification therapeutic regimen in patients with probable chronic migraine and probable medication overuse headache. J Headache Pain. 2010;6:334-7.

42. Zidverc-Trajkovic J, Pekmezovic T, Jovanovic Z, Pavlovic A, Mijajlovic M, Radojicic A, et al. Medication overuse headache: clinical features predicting treatment outcome at 1-year follow-up. Cephalalgia. 2007;27:1219-25.

43. Baumgartner C, Wessely P, Bingöl C, Maly J, Holzner F. Long-term prognosis of analgesic withdrawal in patients with drug-induced headache. Headache. 1989;29:510-4.

44. Katsarava Z, Muessig M, Dzagnidze A, Fritsche G, Diener H, Limmroth V. Medication overuse headache: rates and predictors for relapse in a 4-year prospective study. Cephalalgia. 2005;25:12-5.

45. Hagen K, Albretsen C, Vilming ST, Salvesen R, Grønning M, Helde G, et al. A 4-year follow-up of patients with medication-overuse headache previously included in a randomized multicentre study. J Headache Pain. 2011;12:315-22.

46. Fritsche G, Eberl A, Katsarava Z, Limmroth V, Diener HC. Drug-induced headache: long-term follow-up of withdrawal therapy and persistence of drug misuse. Eur Neurol. 2001;45:229-35.

47. Katsarava Z, Limmroth V, Finke M, Diener H, Fritsche G. Rates and predictors for relapse in medication overuse headache: a 1-year prospective Study. Neurology. 2003;60:1682-3.

48. Grazzi L, Andrasik F, D'Amico D, Usai S, Kass S, Bussone G. Disability in chronic migraine patients with medication overuse: treatment effects at 1-year followup. Headache. 2004;44:678-83. 
49. Boe M, Salvesen R, Mygland A. Chronic daily headache with medication overuse: a randomized follow-up by neurologist or PCP. Cephalalgia. 2009;29:855-63.

50. Boe M, Salvesen R, Mygland A. Chronic daily headache with medication overuse: predictors of outcome 1 year after withdrawal therapy. Eur J Neurol. 2009;16:705-12.

51. Rossi P, Faroni J, Nappi G. Medication overuse headache: predictors and rates of relapse in migraine patients with low medical needs. A 1-year prospective study. Cephalalgia. 2008;28:1196-200.

52. Lampl C, Jensen R, Martelletti P, Mitsikostas DD. Refractory headache: one term does not cover all - a statement of the European Headache Federation. J Headache Pain. 2014;15:50. http://www. thejournalofheadacheandpain.com/content/15/1/50.

53. Fritsche G, Frettloh J, Huppe M, Dlugaj M, Matatko N, Gaul C, et al. Prevention of medication overuse in patients with migraine. Pain. 2010;151:404-13.

54. Krymchantowski AV, Barbosa JS. Prednisone as initial treatment of analgesic-induced daily headache. Cephalalgia. 2000;20:107-13.

55. Pageler L, Katsarava Z, Diener HC, Limmroth V. Prednisone versus placebo in withdrawal therapy following medication overuse headache. Cephalalgia.

2008;28:152-6.

56. Farinelli I, Dionisi I, Martelletti P. Rehabilitating chronic migraine complicated by medication overuse headaches: how can we prevent migraine relapse? Intern Emerg Med. 2011;6:23-8.

57. Boe M, Mygland A, Salvesen R. Prednisolone does not reduce withdrawal headache. Neurology. 2007;69:2631.

58. Rabe K, Pageler L, Gaul C, Lampl C, Kraya T, Foerderreuther $\mathrm{S}$, et al. Prednisone for the treatment of withdrawal headache in patients with medication overuse headache: a randomized, double-blind, placebo-controlled study. Cephalalgia. 2013;33:2027.

59. Taghdiri F, Togha M, Razeghi Jahromi S, Paknejad SM. Celecoxib vs prednisone for the treatment of withdrawal headache in patients with medication overuse headache: a randomized, double-blind clinical trial. Headache. 2015;55:128-35.

60. Valguarnera F, Tanganelli P. The efficacy of withdrawal therapy in subjects with chronic daily headache and medication overuse following prophylaxis with topiramate and amitriptyline. Neurol Sci. 2010;31:S175-7.

61. Silberstein S, Diener HC, Lipton R, Goadsby P, Dodick $\mathrm{D}$, Bussone $\mathrm{G}$, et al. Epidemiology, risk factors, and treatment of chronic migraine: a focus on topiramate. Headache. 2008;48:1087-95.

62. Diener H, Bussone G, Van Oene J, Lahaye M, Schwalen $\mathrm{S}$, Goadsby $\mathrm{P}$, et al. Topiramate reduces headache days in chronic migraine: a randomized, double-blind, placebo-controlled study. Cephalalgia. 2007;27:81423.
63. Roceanu A, Antochi F, Bajenaru O. Chronic migraine new treatment options MAEDICA. J Clin Med. 2014;9:401-4.

64. Grazzi L, Usai S. Botulinum toxin A: a new option for treatment of chronic migraine with medication overuse. Neurol Sci. 2014;35 Suppl 1:37-9.

65. Mathew NT, Jaffri SFA. A double-blind comparison of OnabotulinumtoxinA (BOTOX ${ }^{\circledR}$ ) and topiramate (TOPAMAX ${ }^{\circledR}$ ) for the prophylactic treatment of chronic migraine: a pilot study. Headache. 2009;49:1466-78.

66. Cady RK, Schreiber CP, Porter JA, Blumenfeld AM, Farmer KU. A multi-center double-blind pilot comparison of onabotulinumtoxinA and topiramate for the prophylactic treatment of chronic migraine. Headache. 2011;51:21-32.

67. Mathew NT, Frishberg BM, Gawel M, Dimitrova R, Gibson J, Turkel C. Botulinum toxin type A (BOTOX ${ }^{\circledR}$ ) for the prophylactic treatment of chronic daily headache: a randomized, double-blind, placebo-controlled trial. Headache. 2005;45:293-307.

68. Làinez MJ, Freitag FG, Pfeil J, Ascher S, Olson WH, Schwalen S. Time course of adverse events most commonly associated with topiramate for migraine prevention. Eur J Neurol. 2007;14:900-6.

69. Silberstein SD, Stark SR, Lucas SM, Christie SN, Degryse RE, Turkel CC. Botulinum toxin type A for the prophylactic treatment of chronic daily headache: a randomized, double-blind, placebo controlled trial. Mayo Clin Proc. 2005;80:1126-37.

70. $\quad$ Sarchielli P, Messina P, Cupini LM, Tedeschi G, Di Piero V, Livrea $P$, et al. Sodium valproate in migraine without aura and medication overuse headache: a randomized controlled trial. Eur Neuropsychopharmacol. 2014;24:1289-97.

Interesting paper evaluating a prophylactic option for $\mathrm{MOH}$.

71. Fan W, Lv Y, Ying G, Li W, Zhou J. Pilot study of amitriptyline in the prophylactic treatment of medication-overuse headache: a 1-year follow-up. Pain Med. 2014;15:1803-10.

72. Couch JR, Lenaerts ME. Medication overuse headache: clinical features, pathogenesis and management. Drug Dev Res. 2007;68:1-12.

73. Lenaerts ME, Couch JR. Medication overuse headache. Minerva Med. 2007;98:221-31.

74. Durham PL, Cady R. Insights into the mechanism of onabotulinumtoxinA in chronic migraine. Headache. 2011;51:1573-7.

75. Blumenfeld A, Silberstein SD, Dodick DW, Aurora SK, Turkel CC, Binder WJ. Method of injection of onabotulinumtoxinA for chronic migraine: a safe, welltolerated, and effective treatment paradigm based on the PREEMPT clinical program. Headache. 2010;50:1406-18.

76. Diener H, Dodick D, Aurora S, Turkel C, Degryse R, Lipton R, et al. OnabotulinumtoxinA for treatment of chronic migraine: results from the double-blind, randomized, placebo-controlled phase of the Preempt 2 trial. Cephalalgia. 2010;30:804-14. 
77. Bendtsen L, Evers S, Linde M, Mitsikostas DD, Sandrini $\mathrm{G}$, Schoenen J. EFNS guideline on the treatment of tension-type headache - report of an EFNS task force. Eur J Neurol. 2010;17:1318-25.

78. Estemalik E, Tepper S. Preventive treatment in migraine and the new US guidelines. Neuropsychiatr Dis Treat. 2013;9:709-20.

79. Lipton RB, Silberstein SD. Episodic and chronic migraine headache: breaking down barriers to optimal treatment and prevention. Headache. 2015;55 Suppl 2:103-22.

80. Rokyta R, Fricová J. Neurostimulation methods in the treatment of chronic pain. Physiol Res. 2012;61 Suppl 2:S23-31.
81. Martelletti P, Jensen RH, Antal A, Arcioni R, Brighina $\mathrm{F}$, de Tommaso $\mathrm{M}$, et al.

Neuromodulation of chronic headaches: position statement from the European Headache Federation. J Headache Pain. 2013;14:86.

82. Arcioni R, Palmisani S, Mercieri M, Vano V, Tigano S, Smith T, et al. Cervical $10 \mathrm{kHz}$ spinal cord stimulation in the management of chronic, medically refractory migraine: a prospective, open-label, exploratory study. Eur J Pain. 2015. doi:10.1002/ejp.692.

83. Cicolini G, Palma E, Tafuri E, Sansoni J,

Giamberardino MA. Effectiveness of the telephoniccase-management for treatment of headache. A pilot study. Prof Inferm. 2011;64:173-8. 